\title{
Development of Digital Real-time Remote Training Demonstration Device
}

\author{
Yi Yongfei ${ }^{1, a}$, Zhu Yuanda ${ }^{1, a}$, Zhan Hengfu ${ }^{2, b}$, Zhu Shaohong $^{3, c}$, Gao Tiejun ${ }^{3, c}$ \\ ${ }^{1}$ Liaoning Skill Training Center \\ ${ }^{2}$ Benxi Power Supply Company, State Grid Liaoning Electric Power Supply Co., Ltd. \\ ${ }^{3}$ Tieling Power Supply Company, State Grid Liaoning Electric Power Supply Co., Ltd. \\ a22181221@qq.com, b bxwl_650928@126.com, ${ }^{\mathrm{c}}$ 40136962@qq.com
}

Keywords: Multi-media; Wireless Transmission; Real-time Transmission

\begin{abstract}
Real-time display system for remote training consists of video recorder with wireless transmission module, relay router, display terminal and video recorder carrier with multiple wireless transmission modules. The display terminal includes projector, desktop, tablet computer or smart phones. The video recorder carrier is the shoulder girdle. A bag is set at the front of the shoulder girdle for the embedding of video recorder. The bag mentioned also has the transparent or hollowed-out window for the camera of the corresponding video recorder and the matrix-like through-hole for the microphone of video recorder. Such design can facilitate teaching, enhance the teaching effect and guarantee the fairness and justice of competition.
\end{abstract}

\section{Introduction}

Along with the rapid development of modern technology and people's increasing reliance on modern technological products, the cable communication technology and means that are currently widely applied have failed to met the demands of people who are possessed of rich imagination. Mobile communication has become popular in people's life, thus advancing the research and development as well as the launching of new technology. The wide application of WiFi technology and the advent of $3 \mathrm{G}$ times in particular have provided new and efficient channel for the mobile audio and video data transmission. To meet the increasing social demands, it is critical to fully capitalize on the transmission broadband of wireless channel and the wireless transmission technology of high-definition network data. In the training and teaching of electric power application, the location of the electrical equipment has limited the teaching effect. Consequently, the judges are unable to observe some of the operational steps and therefore cannot objectively correct or evaluate the proficiency of the operators.

\section{Major Design}

\section{Image Monitoring Transmission System of Fixed Point}

Wireless image transmission system can be divided into two categories from the perspective of application: one is the image monitoring transmission system of fixed point and the other is the mobile video image transmission system.

The wireless image monitoring transmission system of fixed points is mainly applied to the places where the cable closed-circuit monitoring system is difficult to be realized, such as the monitoring system of the port wharf, the video and data monitoring of river and water conservancy project, forest fire prevention monitoring system and urban security monitoring.

\section{Mobile Video and Image Transmission System}

Besides the demands for the image monitoring of fixed points, mobile image transmission is also in great need. The mobile video and image transmission has been widely applied to the public security command vehicles, vehicle for the exploration of traffic accidents, vehicles for the scene command by fire soldiers, customs, oilfield, mine, water conservancy, power, finance, maritime 
affairs and other emergencies and emergency command system with the role of transmitting the real-time images to the command center. As a result, the decision-makers of the command center might feel on the scene, thus enhancing the accuracy and instantaneity of decisions as well as the working efficiency.

\section{WIFI Wireless Network Camera}

WIFI wireless network camera requires coordination with the wireless router, which should be connected with the internet. If the WIFI wireless network camera is used, the advantage is that it does not require wiring; therefore, it is quite convenient to install and add cameras with low cost. However, the disadvantage is that the camera can be put into normal usage only when the simple network configuration is conducted according to the specification; the distance between the network camera and the wireless router shall be controlled within 30 to 100 meters. Any excess of this range requires the specialized antenna or nearby installment of wireless router. At the same time, the operating frequency of the wireless router can be set in places where the wireless router is intensively used.

The channel is divided into several component channels to deliver audio, video and digital signals, which are therefore called broadband transmission. Broadband is the frequency band that is larger than the audio bandwidth, which includes most of the electromagnetic Spectrum. Such system with wide band transmission is called the broadband transmission system that can decompose the link capacity into two or more channels. Each channel can carry different signals, which is the broadband transmission.All the channels in broadband transmission can send off signals, such as CATV and ISDN. The width of frequency band is $>=128 \mathrm{kbps}$.

Generally speaking, compared with baseband transmission, broadband has the following advantages:

It allows the transmission of audio image and data information in the same channel, giving the system diverse functions;A broadband channel can be divided into many logic baseband channel that allows multiplexing; therefore, the capacity of channel has been considerably increased;

The distance supported by broadband transmission is longer than that of the baseband because the digital signal is sent through baseband transmission. In spite of the high transmission rate of digital signal, the higher transmission rate, however, is accompanied with shorter transmission distance; a broadband channel can be divided into many logical channels (In the division of broadband channel, the controllable division still remains as only one data line instead of multiple lines after division), thus ensuring the transmission of various audio, image and digital information in the same physical channel. The data are transmitted in multiple logical channels through different signals (analog signal and digital signal).

In the training and teaching in the field of electric power application, the location of electrical equipment has resulted in the not ideal teaching effect. As a result, some judges are unable to have a clear look at some of the operation details, thus failing to objectively correct or evaluate the operational level of the operators. For example, in the tower practical teaching, due to the height of tower and large number of students, most of the students do not have opportunities to ascend to the top of the tower to observe the demonstration of the teachers. However, as the result of the limited training time, it is impossible for teachers to offer repeated demonstration to each of the student. In the teaching of the transformer equipment operating mechanism secondary circuit, limited by the equipment space, only 2 to 3 students standing near the teacher can directly see the operational details of the teachers, a fact that results in the decreasing learning interest of the students and poor effect, affecting the training consistency and influencing the training progress. The students also say that they cannot see and listen clearly and complain about the poor training effect. In actual assessment or competition, as the judges cannot clearly observe the operation details, they cannot offer an objective correction or evaluation of the proficiency of the operators.

To address the aforesaid issues, this equipment offers a distant real-time display system, which can facilitate teaching and markedly enhance the teaching effect, thus guaranteeing the competition fairness and justice of assessment and competition. 
Real-time display system for remote training consists of video recorder with wireless transmission module, relay router, display terminal and video recorder carrier with multiple wireless transmission modules. The display terminal includes projector, desktop, tablet computer or smart phones. The video recorder carrier is the shoulder girdle. A bag is set at the front of the shoulder girdle for the embedding of video recorder. The bag mentioned also has the transparent or hollowed-out window for the camera of the corresponding video recorder and the matrix-like through-hole for the microphone of video recorder.

In the aforesaid remote practical training real-time display system, the lock catch is set on the top of bag of shoulder girdle so as to prevent the falling of the video recorder when the teacher is offering the demonstration activities.

System development platform. The whole module (system) is developed on the basis of the SOC hardware platform. By means of real-time nested A_LINUX and the optimized H, 264 image compression algorithm, the video and audio simulated electricity- $\operatorname{Tr} \mathrm{N}$ is converted into digital signal through AI)C and sent to ASOC for the collection and compression through SOC. Then the compressed code stream is sent to PC through network. PC software is based on Microsoft operating system. The main program adopts the $\mathrm{C}++$ language and decompresses for reverted display so as to offer real-time monitoring.

The realization of video and audio chatting system includes the collection, compression, transmission, decompression and playing of audio and video signal. The system takes the ordinary PC as the terminal and adopts microphone and video camera to realize the collection of audio and video signal and simply the software is adopted for the compression, decompression storage and playing of video and audio signals. The instantaneity of audio and video is more important than reliability. Therefore, the real-time transmission contract (RTC) is adopted for transmission. The decompressed video data is encapsulated and sent to the receiver through UDP contract. At the same time, the decoding is played back at the receiver.

Before the application program is connected with it, it can properly handle the event of real-time communication fire off during the session. In PC-PC communication, the application program captures volume level, media, client-end information and changes in session state. As suggested by the code below, an event filter is established to capture the specific RTC event type.

IEventMask sets a group of events that the application programs are interested in. CRTCEvents send events to the affiliated client. The object of RICEvents also establishes an interface between the application program and IRTCEventNotification. All the real-time communication events are processed by RTVEvents.

\section{Conclusion}

The video recorder sends the video and audio signal to the relay router through wireless transmission module and the relay router also sends the video and audio signal to the wireless receiving module of display terminal. The relevant personnel can see the demonstration through the display terminal and listen to their explanation. The real-time demonstration of remote practical training ensures that the personnel who are far away from the demonstration site can simultaneously see the whole demonstration process. What's more, it can also facilitate teaching, assessment or competition, thus reaching the ideal effect.

\section{References}

[1] Zhou Jian, Dai Meie, Yu Zhenjian. Adaptive Technology of Remote Real-time Video Transmission [J], Tsinghua University (Natural Science), 2004, 44 (07): 966-968.doi: 10.3321 / j.issn: $1000-0054.2004 .07 .027$.

[2] Zhang Qin, He Wei, Li Qian. 3G-based Dual-mode Remote Video Monitoring System [J]. TV technology, 2009, 33(08): 95-98 .doi: 10.3969 / j.issn.1002-8692.2009. 08.045. 
[3] Li Xiaowen, Li Guiyong, Chen Xianliang. TD-SCDMA Third Generation Mobile Communication System, signaling and implementation [M], Beijing: People's Posts and Telecommunications Press, 2003.

[4] Yang Dacheng CDMA20001x Mobile Communication System [M] Beijing: Mechanical Industry Press, 2003.

[5] Li Ping, Liu Ke. Research on User-oriented Access Control Strategy in Video Monitoring System [J], Computer Engineering and Application, 2008,26 (10):.. 2716-2718 .doi: 10.3969 / j.issn.1000-7024.2005.10.048 